\title{
Diagnóstico Territorial Integral del municipio de Ciudad Darío ${ }^{1}$
}

\author{
Laurent Dietsch ${ }^{1}$ y Edwin Novoa ${ }^{2}$ \\ ${ }^{1}$ Universidad Centroamericana, Área de Desarrollo Agrario y Agropecuario (ADAA), correo electrónico: ldietsch@cablenet.com.ni \\ 2Universidad Centroamericana, Instituto de Acción Social “Juan XXIII”, correo electrónico: ednovoa@ns.uca.edu.ni
}

Recibido: septiembre de 2009 / Aceptado: julio de 2010

EN ESTE ARTÍCULO SE PRESENTA EL "DIAGNÓSTICO TERRITORIAL INTEGRAL de Ciudad Darío" realizado como trabajo de fin de curso de la Maestría en Desarrollo Rural de la Universidad Centroamericana. Este estudio ha buscado contribuir a la formulación de propuestas de intervención de los actores sociales del municipio de Ciudad Darío sobre los procesos estratégicos de desarrollo del territorio. Se realizó una zonificación integral del municipio, identificándose cuatro zonas: una zona alta, campesina de agricultura de subsistencia; una zona de laderas secas; una planicie seca de latifundio ganadero; y finalmente, una zona de llanos y vegas fértiles de riego semi-intensivo. Posteriormente, se identificaron y clasificaron por capital (ambiental, económico, social-humano y político-institucional) y nivel territorial, los principales procesos de cambio que afectan o podrían afectar al municipio. Su análisis permitió caracterizar las principales oportunidades y amenazas para el desarrollo del territorio y, al relacionarlo con las fortalezas y debilidades identificadas en la zonificación, evidenciar los principales factores que podrían incidir en el municipio y sus principales retos. Para enfrentar estos retos, se identificaron tres ejes estratégicos: la reducción de los niveles de inseguridad alimentaria y vulnerabilidad ambiental del municipio; el fomento integral de las cadenas de producción de hortalizas; y la prevención de riesgos sociales. Para cada uno de estos ejes se ha definido un conjunto de acciones ordenadas por capital y nivel territorial, orientadas a incidir sobre los principales procesos de cambio identificados. Finalmente, se definieron ejes estratégicos transversales enfocados al desarrollo de capacidades de incidir sobre los procesos claves de desarrollo del municipio.

Palabras clave: Desarrollo rural sostenible/enfoque territorial/análisis sistémico

\section{Introducción}

El municipio de Ciudad Darío está ubicado a $80 \mathrm{~km}$. al Norte de la capital, Managua. Refleja típicamente la problemática del trópico seco de pendiente de Nicaragua, para el cual no existe todavía una propuesta de desarrollo consistente. La Universidad Centroamericana tiene varios años de estar interviniendo en este territorio a través de varios programas y/o instituciones: el Instituto de Acción Social "Juan XXIII" y el Programa de Desarrollo Agrario y Rural. 


\section{Objetivos}

\section{Objetivo general:}

Realizar un diagnóstico territorial integral del municipio de Ciudad Darío para contribuir a la formulación de propuestas de intervención de los actores sociales del mismo sobre los procesos estratégicos de desarrollo de su territorio.

\section{Objetivos específicos:}

a. Realizar una zonificación integral del municipio basado en el análisis de los capitales territoriales (ambiental, económico, social-humano y político-institucional).

b. Identificar y analizar los procesos de cambio claves en el territorio, los actores de estos procesos y sus interacciones.

c. Realizar propuestas de intervención conjuntas de los actores sociales para incidir sobre estos procesos en los campos y niveles territoriales pertinentes de tal forma que contribuyan más eficientemente al desarrollo integral y sostenible del municipio.

\section{Desarrollo del trabajo}

\subsection{Las principales bases teóricas del estudio}

En el marco de esta investigación, concebimos el desarrollo rural sostenible como un "proceso de transformación de las sociedades rurales y sus unidades territoriales, centradas en las personas, participativo, con políticas específicas dirigidas a la superación de los desequilibrios sociales, económicos, institucionales, ecológicos y de género, a la reducción de la pobreza rural que busca ampliar las oportunidades y fortalecer las capacidades de los actores locales para el desarrollo humano, en el contexto de un sistema social ajustado a las necesidades básicas y a los deseos de los individuos y los grupos sociales que lo componen" (Dietsch, Novoa \& Picado, 2006). Todo ello desde una perspectiva integral que revaloriza las funciones del medio rural, reconociendo que sólo con la integración del conjunto urbanorural se puede propiciar el desarrollo y superar visiones sectorizadas.

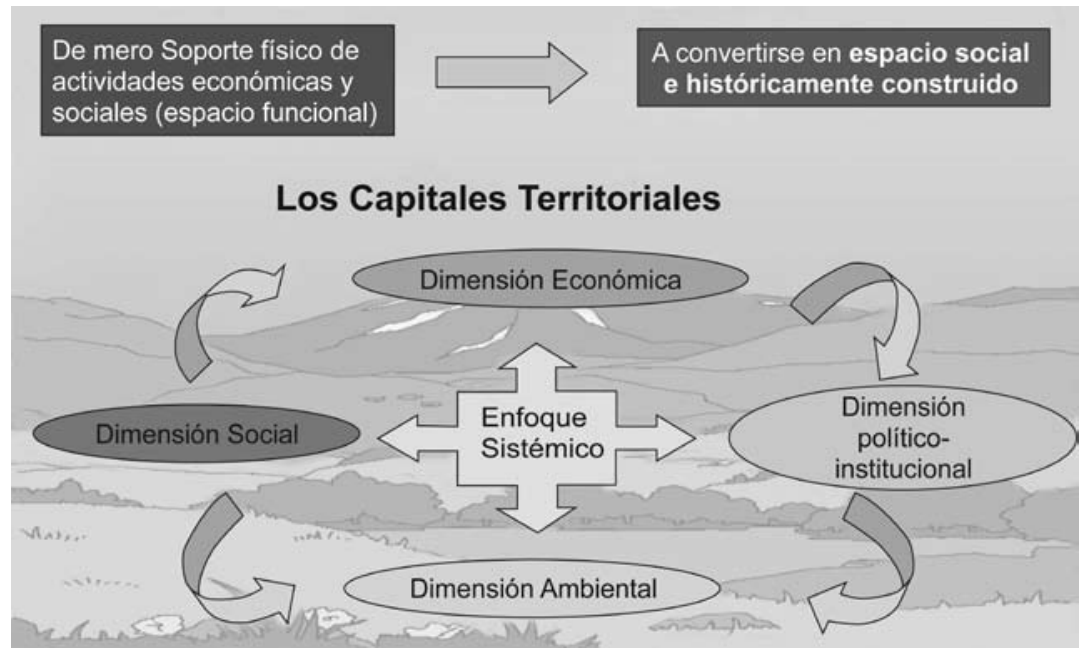

Ilustración 2. El territorio desde un enfoque sistémico 
Consideramos el territorio como un espacio multidimensional que no es mero soporte físico de las actividades humanas, sino un sistema complejo donde interactúan factores económicos, sociales, ambientales, políticos y culturales (Cordero-Salas, Chavarría, Echeverri, \& Sepúlveda, 2003). El enfoque sistémico implica abordar los procesos de desarrollo de un territorio desde la perspectiva de todos los elementos que lo conforman en interacción con su entorno, considerando la interacción de sus múltiples dimensiones (económicas, sociales, ambientales, humanas, etc.) (IICA, 2003)

Un territorio es atravesado por múltiples procesos de cambio, de naturaleza y de alcance geográfico diferentes, con implicaciones diversas para su dinámica global. Algunos tendrán efectos positivos sobre el desarrollo del territorio y otros, efectos negativos. Estas dinámicas provocan cambios en los territorios, tanto en su estructura como en las interrelaciones existentes. Estos cambios se dan en el tiempo y de forma diferente, según el lugar donde se suceden. En ese sentido, el análisis de las dimensiones temporales y espaciales de los procesos es clave para analizar las dinámicas territoriales ya que, como consecuencia de ello, una misma problemática de desarrollo se puede plantear de diversas maneras y requerir acciones diferenciadas pero complementarias en los diferentes niveles territoriales.

Sobre esta base, promover el desarrollo territorial sostenible del municipio implica incidir sobre estos procesos de manera que favorezcan aquellos cambios vistos como "positivos", en términos de desarrollo, por los actores locales, y/o frenar o revertir aquellos cambios considerados como "negativos". Sin embargo, estos procesos son múltiples y multidimensionales, de tal forma que no podemos pretender incidir sobre todos, sino solamente sobre aquellos más relevantes para el desarrollo sostenible del territorio.

Por lo tanto, la identificación de procesos claves debe surgir a través de un análisis, de un reconocimiento de prioridades negociadas y decididas por los actores del territorio. Estos son criterios fundamentales para la identificación de dichos procesos. Por considerarlos pertinentes y estratégicos, se necesita incidir en ellos para generar cambios dinamizadores que impulsen el desarrollo del territorio y que generen externalidades positivas en su conjunto.

Finalmente, sabiendo que un territorio es un sistema que alberga una variedad de actores con múltiples intereses y que existen diferentes niveles territoriales, se debe actuar de manera coherente y complementaria en los diferentes niveles en base a una adecuada identificación de los intereses y motivaciones básicas de los actores (Gutiérrez, 2005), así como de una sólida caracterización de los procesos, sus límites e implicaciones en los distintos niveles territoriales.

Coincidimos con el planteamiento de que "el espacio geográfico está hecho de superposiciones, de interferencias más que de límites" (Touzard, citado por Groppo, 2003, p. 9). En un mismo espacio geográfico pueden coexistir y superponerse distintos territorios en función de la visión que los actores sociales tengan y de la problemática identificada.

Por consiguiente, en Ciudad Darío identificamos y ordenamos las propuestas de intervención a partir de cuatro capitales (ambiental, económico, social-humano y político-institucional) 
y estructurados según los niveles pertinentes de intervención. Partimos del análisis de los capitales territoriales para entender la dinámica de "funcionamiento" del territorio en su doble dimensión geográfica e histórica, como base para la realización de un análisis FODA del territorio y la formulación de líneas estratégicas de desarrollo.

\subsection{Los principales pasos del proceso de desarrollo}

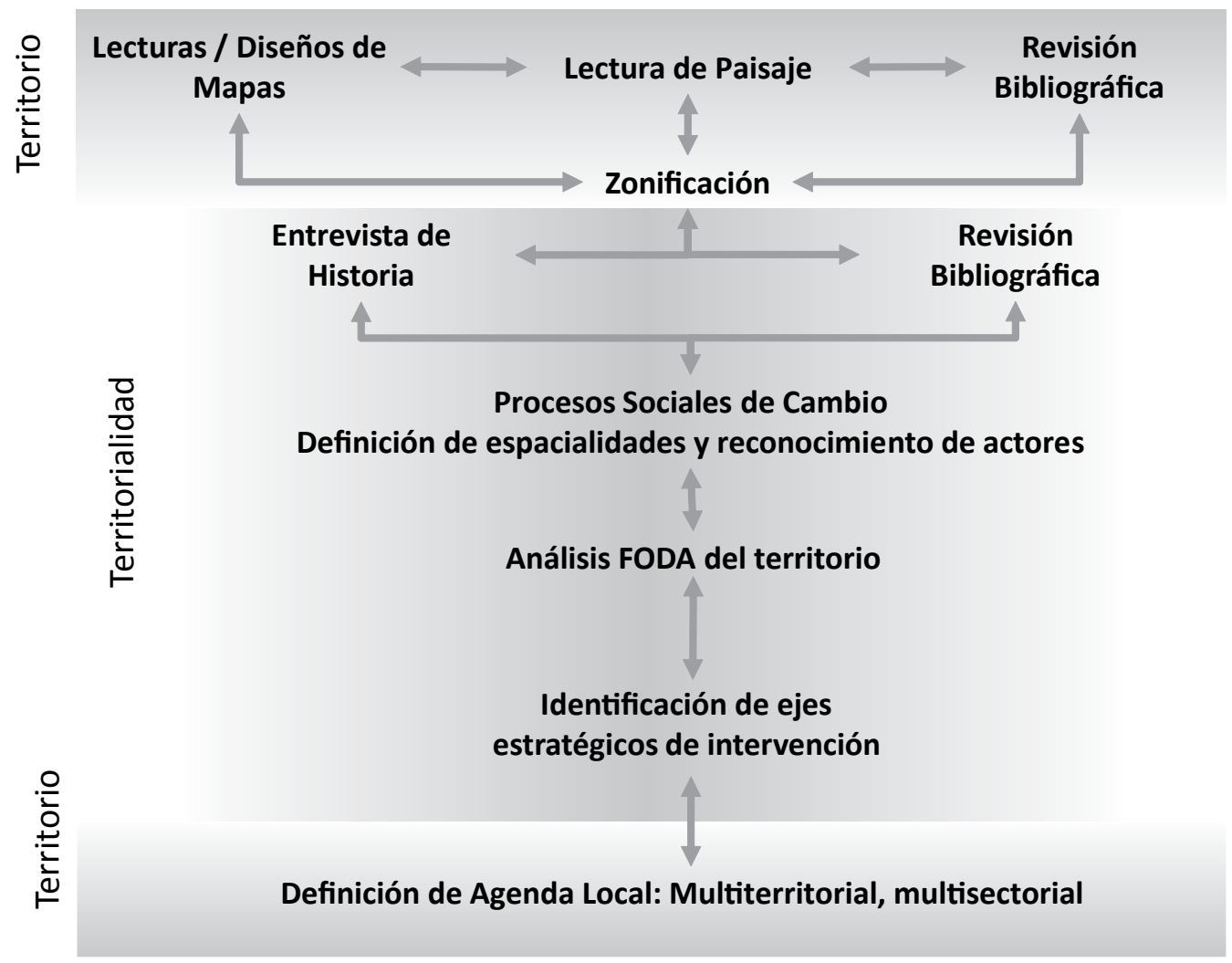

Ilustración 3. Etapas de metodología de análisis de sistema territorial

1.- Zonificación general de los capitales territoriales basado en cuatro capitales: ambiental, económico, social-humano y político-institucional.

Los principales productos obtenidos en esta etapa fueron los siguientes:

- Primera síntesis con la caracterización de los diferentes componentes de los capitales territoriales.

- Zonificación agro socioeconómica del municipio.

- Identificación de las principales fortalezas y debilidades en las diferentes zonas identificadas. 
Se realizó un análisis de los cuatro capitales territoriales para conocer sus principales características y estructuración en el municipio. Se utilizó información secundaria, lecturas y análisis de mapas territoriales, realización de transeptos, observación de paisajes y entrevistas a informantes claves en el territorio.

2.- Identificación y profundización del análisis de los procesos claves en el territorio, los actores de estos procesos y sus interacciones.

Sobre la base de recolección de información secundaria (Nitlapan-UCA: 2005) y entrevistas a informantes claves, conocedores de las dominantes históricas del municipio, se procedió a la identificación y caracterización de los principales procesos de cambio que afectan o afectarán al territorio. Luego, se profundizó en su análisis para obtener los siguientes elementos:

- Identificación, por capital territorial, de los principales procesos de cambio que lo afectan o afectarán (positiva o negativamente).

- Análisis de la dinámica histórica de cada uno de los procesos de cambio identificados y delimitación de los niveles territoriales sobre los cuales se desarrollan.

- Identificación y caracterización del encadenamiento de estos procesos de cambio (causas y consecuencias en el mismo capital pero también en los demás capitales territoriales).

- Identificación de los actores de estos procesos y sus interacciones.

3.- Análisis FODA del territorio y formulación de líneas estratégicas de desarrollo

En base al análisis realizado, se formularon propuestas de líneas estratégicas de desarrollo para incidir sobre estos procesos de cambio en sus distintos niveles territoriales, siguiendo los pasos señalados a continuación:

- Realización de un FODA con los principales elementos obtenidos en el proceso de análisis territorial.

- Integración de los diferentes elementos priorizados en el FODA para formular propuestas de intervención sobre los procesos claves de cambio del territorio, aprovechando oportunidades identificadas y/o limitando el impacto de las amenazas, basándose sobre las fortalezas identificadas y/o buscando cómo superar las limitantes existentes.

- Para cada proceso se definieron y ordenaron por niveles y capitales territoriales las líneas de acción, ya que se considera que solamente con un conjunto de acciones articuladas en diferentes niveles y campos se puede intervenir de manera efectiva sobre determinados procesos.

En consecuencia, estas acciones y factores fueron ordenados por campo y por nivel territorial en el que se deben de impulsar, tal y como se muestra en la siguiente matriz: 
Cuadro 1. Estructuración de estrategias multi-dimensionales y multi-territoriales

\begin{tabular}{|l|l|l|l|l|}
\hline \multicolumn{1}{|c|}{ Niveles/Campos } & Ambiental & Económico & Humano & $\begin{array}{c}\text { Social y Político- } \\
\text { institucional }\end{array}$ \\
\hline Nacional & & & & \\
\hline Departamental & & & & \\
\hline Intermedio & & & & \\
\hline Municipio & & & & \\
\hline Comunidad & & & & \\
\hline
\end{tabular}

Fuente: Elaboración propia.

Finalmente, se realizó, un taller de retroalimentación y discusión con los principales actores del territorio.

\section{Los principales resultados obtenidos}

48 En base al análisis de los cuatro capitales territoriales de Ciudad Darío, se realizó una zonificación integral del municipio validada por los actores locales, delimitando cuatro zonas, dos dividiéndose en dos sub-zonas diferentes: una zona alta (1), campesina de agricultura de subsistencia; una zona de laderas secas (2), con una sub-zona campesina de subsistencia y otra de minifundio semi-proletarizado; una zona planicie seca de latifundio ganadero (3); y, finalmente, una zona de llanos y vegas fértiles de riego semi-intensivo (4), con una sub zona campesina y otra empresarial.

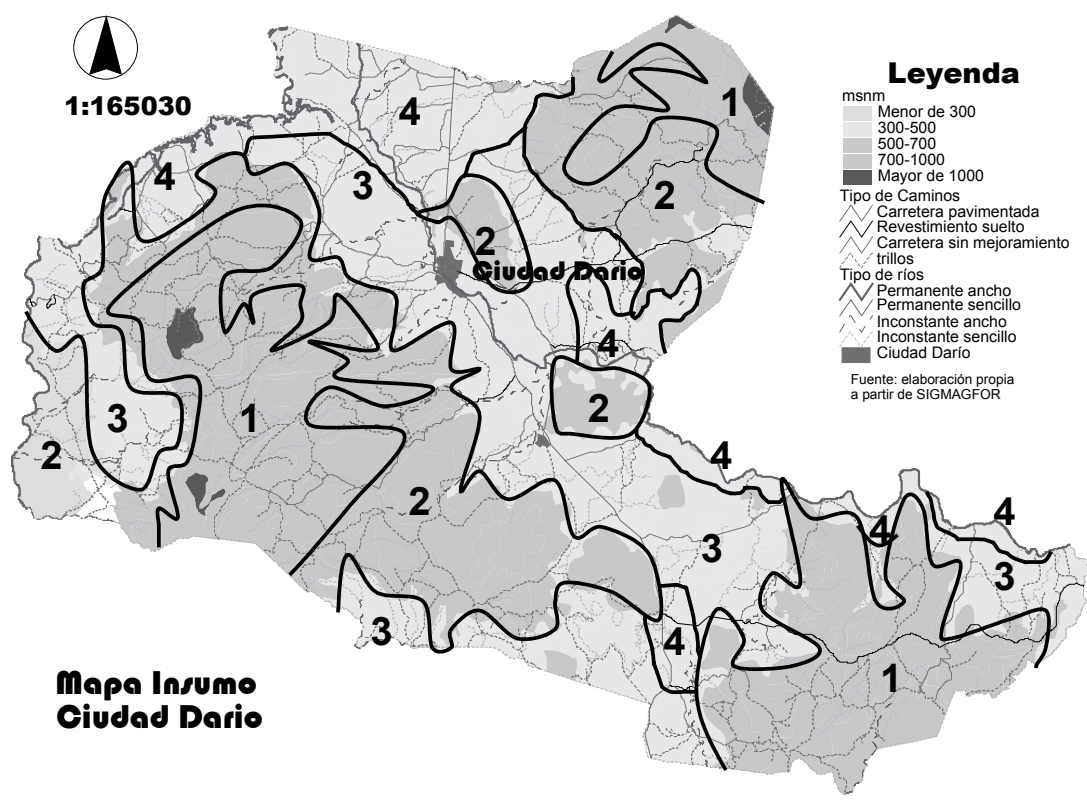

Ilustración 4. Mapa zonificación Ciudad Darío 
Los factores más relevantes para diferenciar las zonas fueron: la topografía, con su incidencia directa sobre las diferenciaciones climáticas y sobre los suelos; el acceso al agua (subterránea o superficial), particularmente relevante en un contexto de trópico seco; las vías de acceso; y finalmente, la estructura de la tenencia de la tierra.

El análisis de las diferentes zonas permitió identificar las principales fortalezas y debilidades del municipio.

Cuadro 2. Principales fortalezas y debilidades del municipio de Ciudad Darío

\begin{tabular}{|c|c|}
\hline Fortalezas & Debilidades \\
\hline $\begin{array}{l}\text { Ventajosa ubicación geográfica del } \\
\text { municipio: atravesado por la carretera más } \\
\text { importante del país y cercano a mercados } \\
\text { nacionales e internacionales. } \\
\text { Uso de suelos y experiencia de actores } \\
\text { económicos: suelos con muy buena calidad } \\
\text { y acceso a riego (zona 4); mayor proporción } \\
\text { de suelos aptos para desarrollo forestal } \\
\text { y ganadero (zonas } 1 \text {, } 2 \text { y } 3 \text { ); experiencia } \\
\text { productiva en la ganadería, hortalizas, } \\
\text { producción de ladrillos de barro y actividad } \\
\text { "liniera" } 2 \text { como principal fuente de ingresos } \\
\text { de habitantes del municipio. } \\
\text { Desarrollo de procesos organizativos } \\
\text { a nivel local (comités comunitarios, } \\
\text { asociación de productores, cooperativas de } \\
\text { servicios, etc.) y presencia de organismos } \\
\text { gubernamentales y no gubernamentales. }\end{array}$ & $\begin{array}{l}\text { Ubicación del municipio en las zonas más } \\
\text { secas del país con suelos degradados y } \\
\text { erosionados. Esto se suma a niveles altos de } \\
\text { inseguridad sobre la tenencia y asimetría en } \\
\text { el mercado de la tierra. } \\
\text { Difícil disponibilidad y acceso a agua de } \\
\text { calidad para el consumo y uso productivo; } \\
\text { dependencia del valle de Sébaco para el } \\
\text { aprovisionamiento de agua potable para el } \\
\text { casco urbano } \\
\text { Predominancia delógicas tradicionales de } \\
\text { producción: lógicas extensivas y extractivas } \\
\text { de producción (ganaderos); uso alto de } \\
\text { químicos y lógica de "lotería" (hortaliceros); } \\
\text { alta dependencia de cultivos anuales por } \\
\text { parte de la población campesina; ladrilleros } \\
\text { concentrados sobre mercados tradicionales } \\
\text { El sector liniero poco articulado con los } \\
\text { demás sectores económicos delmunicipio, } \\
\text { por lo cual, no juega papel de "motor" del } \\
\text { desarrollo local, lo que refleja un bajo nivel } \\
\text { y capacidad de acción colectiva. }\end{array}$ \\
\hline
\end{tabular}

Se identificaron los procesos de cambio del municipio considerados claves para el presente y futuro del mismo. La mayoría de estos procesos son muy complejos ya que afectan los diferentes capitales territoriales y tienen repercusiones en diferentes niveles territoriales.

Para facilitar de manera operativa su estudio y posterior definición de estrategias de intervención, estos procesos han sido analizados desde cada uno de los capitales territoriales y por los niveles territoriales en los cuales se desarrollan, sin perder de vista sus articulaciones con los demás capitales y niveles territoriales. Se identificaron los siguientes procesos: 
- En lo ambiental

- A nivel local, aumento de vulnerabilidad de las poblaciones ubicadas en las microcuencas del municipio, pérdida de áreas de hortalizas por los campesinos y semi campesinos de las zonas altas y de laderas, y migración de población hacia el casco urbano y el exterior del país.

- A nivel municipal, reducción de cobertura vegetal vinculada a la ampliación de áreas para la agricultura de subsistencia y la extracción de leña por los semi-campesinos y campesinos extensivos, de manera articulada con el desarrollo de la ganadería por los medianos y grandes finqueros extensivos, y la demanda energética del sector ladrillero del municipio.

- A nivel departamental, pérdida de cobertura boscosa y contaminación de ríos en la cuenca del Río Grande, provocando una pérdida del potencial de riego en las vegas del municipio tanto para los empresarios como para los campesinos y semicampesinos intensivos.

- A nivel global, los procesos de cambio climático acentuarán los niveles de vulnerabilidad y de inseguridad alimentaria en el municipio. Así mismo, podrían propiciar sistemas de pago por servicios ambientales.

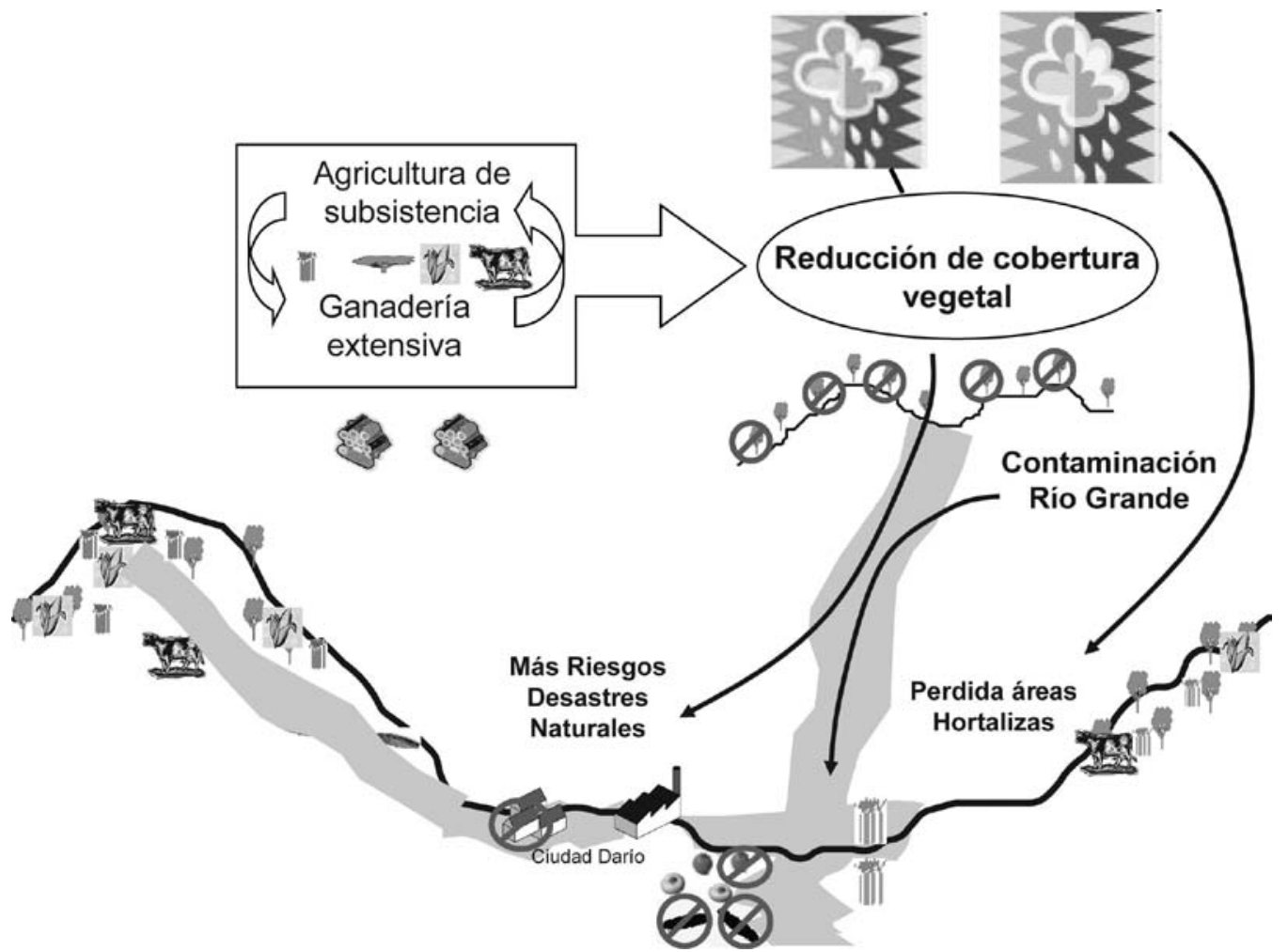

Ilustración 5. Procesos de cambio ambiental en Ciudad Darío 
- En lo económico

- A nivel local, el desarrollo de la "liniería" ha convertido esta actividad en la primera fuente de ingresos del municipio y explica la importancia del crecimiento urbano de Ciudad Darío en los últimos 10 años.

- A nivel departamental, el desarrollo de las zonas francas generadoras de empleos, aunque mal remunerados, ha provocado un proceso importante de proletarización de poblaciones rurales del municipio y urbanización de la cultura laboral.

- A nivel nacional, la expansión del sector turístico genera oportunidades de desarrollo para el municipio por la demanda de materiales de construcción producidos localmente (ladrillos, tejas, "ranchos típicos", etc.), pero también puede generar amenazas de mayor presión sobre los recursos naturales (leña y barro).

- A nivel regional, el CAFTA podría provocar bajas de rentabilidad de productos tradicionales del municipio (granos básicos, leche, etc.), pero a la vez, crear potencialidades para otros (hortalizas).

- A nivel mundial, la volatilidad del sistema económico (crisis del petróleo, precios de los alimentos, crisis financiera, etc.) impactará en el incremento de los costos de productos y servicios, acentuando las dificultades de seguridad alimentaria, disminuyendo la rentabilidad de la agricultura convencional. Por el contrario, podrá crear oportunidades para la producción de bioenergía y la producción orgánica.

- En lo humano

- Los procesos locales y regionales de migración están reconfigurando fuertemente el municipio tanto en lo económico como en lo social-humano.

- Los procesos de globalización inciden sobre las identidades y patrones culturales así como sobre el desarrollo de riesgos sociales (inseguridad, tráfico de drogas, etc.).

- En lo social y político-institucional

- A nivel local, la emergencia de una variedad de organizaciones locales y la apertura de espacios de concertación público-privados (CDM) aún poco aprovechados, posibilitan la acción colectiva.

- A nivel departamental, diversas pero incipientes iniciativas de acción colectiva, posibilitan la incidencia en este nivel territorial intermedio (conformación de un comité de desarrollo departamental, de una asociación de municipios de la zona seca del departamento de Matagalpa).

- A nivel nacional, los procesos de descentralización están permitiendo espacios de participación ciudadana y de mayores niveles de gestión a nivel local. 
- A nivel global, el proceso de integración regional tiende a facilitar la libre circulación de bienes, servicios y personas, con su impacto tanto en los procesos migratorios como comerciales.

El análisis de estos procesos fue el principal insumo para la identificación de las principales oportunidades y amenazas del territorio.

Cuadro 3. Principales oportunidades y amenazas del municipio de Ciudad Darío

\begin{tabular}{|c|c|}
\hline Op & \\
\hline $\begin{array}{l}\text { Comerciales y productivas: El CAFTA } \\
\text { posibilita mercado de hortalizas, frutas, } \\
\text { etc.; incremento de la demanda de insumos } \\
\text { orgánicos y bio-energéticos por incremento } \\
\text { de precios del petróleo; demanda creciente } \\
\text { de materiales de construcción locales por } \\
\text { desarrollo del sector turístico. } \\
\text { Político-institucionales: Procesos de } \\
\text { descentralización y ley de participación } \\
\text { ciudadana posibilitan apertura de nuevos } \\
\text { espacios de concertación a diferentes } \\
\text { niveles territoriales (Comité de Desarrollo } \\
\text { Departamental, Mancomunidad de } \\
\text { municipios de la zona seca de Matagalpa); } \\
\text { cercanía al Valle de Sébaco, zona priorizada } \\
\text { para el desarrollo agroindustrial. } \\
\text { Ambientales: Con convenciones sobre } \\
\text { cambio climático aumentan posibilidades de } \\
\text { implementar sistemas de pago por servicios } \\
\text { ambientales. }\end{array}$ & $\begin{array}{l}\text { Socio-ambientales: Pérdida de cobertura } \\
\text { boscosa y cambio climático incrementa } \\
\text { vulnerabilidad de la población ante } \\
\text { amenazas de desastres naturales; aumento } \\
\text { de presión sobre recursos naturales como } \\
\text { el bosque (zonas 1 y 2) y el barro para la } \\
\text { fabricación de ladrillos (zona 3); incremento } \\
\text { de niveles de inseguridad alimentaria. } \\
\text { CAFTA afectará seriamente varios } \\
\text { sectores productivos tradicionales del } \\
\text { municipio: arroz, granos básicos, leche, } \\
\text { etc. } \\
\text { Desintegración familiar, ruptura de } \\
\text { tejido social en las comunidades rurales } \\
\text { y pérdidas de capital humano calificado } \\
\text { producto de migraciones y desarrollo zonas } \\
\text { francas por la ausencia de alternativas de } \\
\text { generación de ingresos, principalmente } \\
\text { para los jóvenes. } \\
\text { Aumento de riesgos sociales (prostitución, } \\
\text { narcotráfico, inseguridad) más en las } \\
\text { principales vías de comunicación por ser } \\
\text { ruta de tránsito centroamericana. }\end{array}$ \\
\hline
\end{tabular}

Para enfrentar estos procesos, tomando en cuenta el análisis FODA realizado, se identificaron tres ejes estratégicos de intervención:

1. La reducción de los niveles de inseguridad alimentaria y vulnerabilidad ambiental del municipio.

2. El fomento integral de las cadenas de producción de hortalizas aprovechando la pertenencia parcial del territorio al Valle de Sébaco.

3. La prevención de riesgos sociales provocados por las migraciones, el desarrollo de las zonas francas, así como la apertura de Ciudad Darío a fenómenos regionales 
(tráfico de drogas, explotación sexual infantil, principalmente) por su ubicación sobre la Carretera Panamericana.

Para intervenir en estos ejes es necesario incidir sobre varios procesos de cambio en varios niveles y capitales territoriales, de manera simultánea. Para cada eje se ha definido un conjunto de acciones ordenadas por capital y nivel territorial, constituyendo la base para la definición de acciones colectivas por los actores locales.

Para concluir el proceso se definieron cuatro ejes estratégicos adicionales enfocados al fortalecimiento, de manera transversal, de las capacidades humanas, sociales y políticoinstitucionales de incidencia sobre los procesos claves de desarrollo del municipio:

- Fortalecimiento de capacidades empresariales mediante la consolidación y acompañamiento de grupos locales en el impulso de iniciativas empresariales, centradas sobre rubros potenciales, compatibles con el manejo sostenible de recursos naturales, con efectos dinamizadores en el municipio e integrando jóvenes (hombres y mujeres).

- Fortalecimiento de capacidades de acción colectiva a diferentes niveles mediante la identificación de temas y ejes de interés común a los diferentes niveles y definición de procesos para elaborar estrategias conjuntas; mecanismos para generar mayores niveles de confianza entre los actores; sistema para compartir información transparente y accesible; definición de estrategias concertadas de financiamiento del desarrollo del municipio.

- Disminución de los niveles de inseguridad sobre la tenencia de la tierra y mejoramiento del funcionamiento del mercado de tierra.

- Articulación del desarrollo de la actividad "liniera” con el desarrollo del municipio en su conjunto.

\section{Conclusiones}

La metodología utilizada para el análisis territorial del municipio resultó muy adecuada para el logro de los objetivos propuestos inicialmente, desde el abordaje multidimensional (capitales territoriales) y multi-territorial de un espacio rural (niveles territoriales). Facilitó una rápida identificación de los procesos y actores claves que inciden en la dinámica territorial, así como la formulación y validación de estrategias de desarrollo. Evidenció la importancia de articular las estrategias a través de un conjunto de acciones realizadas en los diferentes niveles territoriales.

La diferenciación del municipio en zonas permitió la identificación y caracterización específica de las principales fortalezas y debilidades de cada una de ellas, para el desarrollo rural sostenible del municipio. De igual manera, el análisis de los procesos claves de cambio, permitió caracterizar las principales oportunidades y amenazas para el desarrollo del territorio que, al analizarlas conjuntamente con las fortalezas y debilidades identificadas en la zonificación, evidenciaron los principales factores que podrían incidir, positiva 0 negativamente, sobre dichos procesos de cambio, así como los principales retos para el futuro del municipio. 
Como resultado de este proceso, se identificaron seis líneas estratégicas de desarrollo para la intervención en igual número de procesos de cambio. Para facilitar procesos de concertación entre los actores locales, recomendamos concretar la acción colectiva alrededor de ejes estratégicos de desarrollo, de manera intersectorial, ordenando la discusión por capitales y niveles territoriales pertinentes.

\section{Notas}

1 Investigación realizada en calidad de trabajo de graduación de la Maestría en Desarrollo Rural de la Universidad Centroamericana en conjunto con Claudio Picado y con asesoría del Msc. Adolfo Hurtado.

2 Liniería: actividad comercial de venta al crédito en el municipio y fuera del mismo, en la cual cada liniero tiene una ruta de distribución con una determinada cantidad de "clientes". De manera periódica (semanal o quincenal), el liniero recorre esta ruta (muchas veces a pie), ofreciendo productos al crédito y cobrando las cuotas generalmente bajas de productos entregados anteriormente

\section{Referencias bibliográficas}

Alcaldía de Ciudad Darío (2001). Plan de Desarrollo Municipio Ciudad Darío. Ciudad Darío, Nicaragua, Documento no publicado.

Cordero-Salas, P., Chavarría, H., Echeverri, R. \& Sepúlveda, S. (2003). Territorios rurales, competitividad y desarrollo. Cuaderno Técnico (23), San José, Costa Rica: IICA.

Dietsch, L., Novoa, E. \& Picado, C. (2006). Diagnóstico territorial integral Ciudad Darío. Trabajo de fin de curso no publicado, Universidad Centroamericana, Managua, Nicaragua.

Groppo, P. (2003). El diagnóstico territorial participativo hacia la mesa de negociación. Orientaciones metodológicas. Borrador para comentarios. FAO.

Gutiérrez, A. (2005). Las prácticas sociales: una introducción a Pierre Bourdieu. Córdoba: Ferreira Editor.

IICA. (2003). El enfoque territorial del desarrollo rural. Coronado, Costa Rica: IICA.

NITLAPAN-UCA (2005). Revisitando el Agro Nicaragüense: Tipología de los sistemas de producción y zonificación agro socioeconómica. Managua: Nitlapan. 